\title{
Hablemos del tiempo. El imaginario publicitario del cambio climático
}

institucional.us.es/ambitos/

\section{Isidro Jiménez Gómez}

Universidad Complutense de Madrid

English Version: Let's talk about time. The imaginary advertising of climate change.

La irrupción en 2007 del cambio climático en el debate público ha venido acompañada de un nuevo marco comunicativo en el ámbito empresarial, la Responsabilidad Social Corporativa, donde la gran empresa se ubica a sí misma como agente clave a la hora de afrontar el reto que supone el calentamiento global. Pero la perspectiva propositiva de este agente de gran relevancia comunicativa recae en la eficiencia energética, el optimismo tecnológico y, sobre todo, en una sociedad de consumo desconectada de los impactos sociales y medioambientales que produce. La desconexión entre el escaparate de la tienda y el patio trasero de la producción, elemento constitutivo de la sociedad de consumo para la gran clase

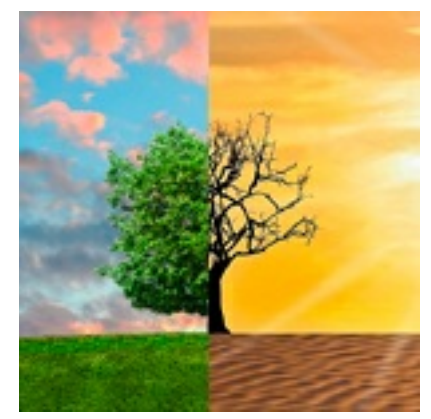
media global, renace en el mito del desacoplamiento absoluto entre el crecimiento económico y sus requerimientos e impactos materiales. Así que es esa idea de eficiencia tecnológica, recurso publicitario constante, el que hoy guía un mito que intenta salvaguardar de forma desesperada la idea de crecimiento económico y consumo para las clases medias.

Palabras clave: Comunicación y Desarrollo, Comunicación y Cambio Social, Sostenibilidad, Tecnología, Publicidad.

Abstract: The emergence of climate change in the public debate in 2007 has been accompanied by a new communicative framework in the corporate sphere, the Corporate Social Responsibility, where the big company places itself as the key agent at the time of facing the challenge that it entails global warming. But the propositive perspective of this agent of great communicative relevance rests on energy efficiency, technological optimism and, above all, on a consumer society disconnected from the social and environmental impacts that it produces. The disconnection between the shop window and the production backyard, a constituent element of consumer society for the large global middle class, is reborn in the myth of the absolute decoupling between economic growth and its requirements and material impacts. So it is this idea of tehcnological efficiency, constant advertising resource, which today guides a myth that tries desperately to safeguard the idea of economic growth and consumption for the middle classes.

Keywords: Communication and Development, Communication and Social Change, Sustainability, Technology, Advertising.

\section{Introducción. Hablemos del tiempo}

Un reciente anuncio de Iberdrola muestra la fotografía de un precioso paisaje nevado y, debajo del todo, una contundente frase: "Líder mundial en la lucha contra el cambio climático". Aunque muchos anunciantes ya nos tienen acostumbrados a este tipo de frases épicas, no deja de sorprender que venga de uno de los grandes representantes del sector energético. Y es que tan solo las diez mayores empresas de este sector son responsables del $70 \%$ de las emisiones de gases de efecto invernadero (GEI) estimadas en España para los sectores fijos en 2015. Es cierto que la contribución de Iberdrola, con 2,6 millones de toneladas de CO2, queda lejos de la empresa líder en emisiones de gases GEI (Endesa, con 33 millones de toneladas de CO2), pero de ahí a que uno de los principales emisores sea "líder mundial en la lucha contra el cambio climático" va mucho trecho. 
Este tipo de campañas, a pesar de todo, reciben premios. Por ejemplo, el periódico El Economista otorgaba hace unas semanas a Iberdrola el Premio Estrella de la Comunicación por, entre otras cosas, "potenciar la cercanía de los mensajes que transmite". Y es que la campaña "Hablemos del tiempo" de Iberdrola utiliza refranes como "hasta el cuarenta de mayo no te quites el sayo" o "por enero florece el romero", reflejo invariable de una tradicional y arraigada preocupación por las variaciones climáticas. Para que estas predicciones meteorológicas continúen cumpliéndose, dice la compañía energética, es necesario cuidar nuestro planeta.

No hay duda de la capacidad de Iberdrola para generar una comunicación cercana, e incluso para ubicarse como uno de los posibles remedios ante el desolador panorama que dibuja el cambio climático, pero esto genera algunos problemas comunicativos. A pesar de ser el causante de dos tercios de las emisiones GEI a nivel global, el sector energético se beneficia de una indudable proyección mediática, en la que la publicidad tiene un papel relevante. Tras un estudio realizado en siete diarios españoles de ámbito nacional, Mercado Saez (2016) sugiere que puede existir un interés de los propios medios por no conectar el cambio climático con el sector energético, "uno de los principales anunciantes en los medios y con mayor relevancia política, económica y social”, explica.

Grandes compañías como Iberdrola han conseguido instaurar un nuevo marco comunicativo en torno a la Responsabilidad Social Corporativa (RSC), espacio clave para la difusión que hacen las empresas de sus acciones en materia medioambiental. Pero avisa Lizcano (2009), la RSC debe también suponer una reestructuración comunicativa, ubicando en el centro a la idea de veracidad. "Si se desea que la responsabilidad social corporativa no sea sólo retórica social corporativa, será necesario que todas las empresas empiecen a determinar con exactitud cuáles son sus emisiones reales, en qué medida influyen en el cambio climático y cómo van a reducir sus emisiones y paliar sus responsabilidades en el cambio climático", explica el informe "Responsabilidad de las grandes empresas energéticas e industriales de España en el cambio climatico 2015" del Observatorio de la Sostenibilidad (OSE, 2015). De hecho, según los datos del Registro Nacional de Emisiones español, no hay mucho espacio para eslóganes optimistas. Las emisiones de gases GEI en 2015 superaron los 339 millones de toneladas equivalentes de CO2, con un aumento de 13,77 millones de toneladas respecto del año anterior. 2015 es el segundo año en que se produce un incremento de las emisiones desde que en 2008 descendieran bruscamente debido a la crisis económica. Estas cifras, explica el Observatorio de la Sostenibilidad, "siguen situando al Estado español a la cabeza de la Unión Europea en el incremento de emisiones desde 1990, año que se toma como referencia para el protocolo de Kioto" (OSE, 2015).

Así que el problema, en principio comunicativo, de la veracidad en las campañas publicitarias que abordan el cambio climático, es también ahora el de si estas campañas pueden suponer, en vez de un impulso en la lucha contra el cambio climático, lo contrario, un freno.

\section{Un spot incómodo}

Tras el éxito de su documental "Una verdad incómoda", el que fuera vicepresidente del gobierno de los EEUU, Al Gore, recibió en 2007 el Premio Príncipe de Asturias y el Premio Nobel de la Paz, este último compartido con el Grupo Intergubernamental de Expertos sobre Cambio Climático (IPCC). A pesar de que el IPCC avisaba desde 1990 de que el Calentamiento Global se estaba acelerando de forma alarmante, su repercusión en los medios de comunicación fue bastante brusca. "De la noche a la mañana un extraño vocablo, IPCC, ha entrado de lleno en los noticiarios, los discursos políticos y la vida de los ciudadanos", decía el periodista Pedro Cáceres (2007). Sin embargo, el cambio climático estaba muy lejos de ser una evidencia. Cuando estrenó "Una verdad incómoda", Al Gore se lamentaba de que, a pesar de ser ya visibles los efectos del cambio climático, "demasiados estadounidenses, políticos y no políticos, siguen sin creer en el calentamiento global" (UIP, 2006). Y ofrecía como prueba un estudio de la revista Science del año 2004 donde se mostraba que, de todos los estudios científicos sobre el calentamiento global publicados en revistas, 928 estaban de acuerdo en que existía y ninguno lo negaba. Sin embargo, examinando las noticias en medios de comunicación, el $53 \%$ de ellas sugería que el calentamiento global no estaba demostrado. "En otras palabras, el mensaje que recibe la gente no concuerda con los hechos", decía AI Gore (UIP, 2006) para enfatizar la importancia de su propio documental. 
Sin embargo, el propio documental estaba formando parte de un punto de inflexión en el tratamiento mediático del cambio climático. A pesar de las controversias, explica Iranzo (2008), el cambio climático se admite oficialmente y se instaura como "una realidad virtualmente incontestable en el lapso de unas breves semanas a comienzos del año 2007". A esta nueva realidad mediática le acompañaba una inevitable reflexión sobre los retos de la humanidad en el futuro y algunos conceptos, como el de sostenibilidad, terminan por ejercer una atractiva pero confusa influencia en las columnas periodísticas de los grandes medios de comunicación. Ahora bien, al debate todavía se unieron otros actores, quizás en parte inesperados pero, en todo caso, esenciales en ese abstracto espacio de influencia discursiva que llamamos la opinión pública. Mientras el gobierno español ponía en marcha una campaña bajo el lema "Te creías el rey de la Creación, no seas el rey de la destrucción", empresas como Acciona, Iberdrola, Endesa, Telefónica, Toyota o BMW apostaban, justo antes de la crisis de 2008, por introducir en su publicidad temáticas como la reducción del gasto energético o la conversión de los residuos en recursos.

Un spot televisivo de Acciona en 2006, emitido tan sólo una semana después del estreno internacional de "Una verdad incómoda", simboliza ese punto álgido. En él, un joven juega con la videoconsola en el salón de un piso compartido cuando su compañero le explica que va a encender el aire acondicionado porque tiene calor. Ante los reproches del primero -“Eso, pon el aire, carguémonos el planeta"- éste se sienta junto a él en el sofá y despliega un argumento antiprimitivista del estilo "¿quieres que volvamos a las cavernas? El anuncio muestra imágenes apocalípticas de carreteras y calles vacías mientras dice la voz en off: "Tendríamos que abandonarlo todo. Diríamos 'no' a todo el progreso conseguido durante siglos y volveríamos a vivir como nuestros antepasados... ¿ ¿ese es el futuro que quieres?". Tras un pequeño pero dramático intervalo, el compañero se incorpora en el sillón y le responde: "Vale. Hagamos lo que dices. Pongamos el aire. Adelante con el progreso. Sigamos exprimiendo los recursos que nos quedan irresponsablemente y ¿sabes lo que pasará? Que se acabarán. Y cuando esto ocurra nos preguntaremos «¿Y qué vamos a hacer ahora sin energía, sin transportes, sin agua corriente, sin nada?» Porque llegado ese día, nada funcionará. $Y$ no hay vuelta atrás. Dejaremos nuestros empleos: ¿de qué nos servirían? ¿No? Y dejaremos las ciudades: ¿qué pintaríamos en ellas? Tendríamos que dejarlo todo. Decir 'no' a todo el progreso conseguido durante siglos y volver a vivir como nuestros antepasados". Y mira a su compañero: “¿Ese es el futuro que quieres?”.

La empresa constructora y energética reivindica así un papel propositivo ante el cambio climático, algo que pronto compartieron los grandes anunciantes. Ezquerra Martínez y Fernández Sánchez (2014) hablan de una nueva tendencia a "relacionar la bondad de un producto con lo ecológico, lo verde que es, o lo cuidadoso que resulta con el medio ambiente". Pero hay más. Las grandes empresas de sectores temáticamente interpelados por el reto de la sostenibilidad medioambiental, como el energético o el automovilístico, han terminado construyendo una narrativa publicitaria ante la crisis medioambiental, describiendo primero el escenario -el retopara terminar cerrando el debate como lo hace cualquier spot: presentar a la compañía y sus productos como parte esencial de la solución.

\section{De la publicidad verde a la sostenibilidad azul}

El 7 de diciembre de 2009 fue otra fecha clave en la reciente historia de la comunicación del cambio climático. Comenzaba en Copenhague una Conferencia de las Naciones Unidas sobre el Clima que pretendía coger el relevo del Protocolo de Kioto. Y entre los distintos anuncios publicitarios que por entonces hacían referencia al tema, destacaba uno cuyo eslogan era, únicamente, "Hopenhague". Esta vez, la campaña no era impulsada por ninguno de los anunciantes habituales sino por la Asociación Internacional de Publicidad (IAA) y una coalición de las principales agencias de publicidad, marketing y medios de comunicación. Así pues, la campaña, que se presentaba como "un movimiento de la gente" lo suficientemente grande como para que "nuestros líderes no lo puedan ignorar" (IAA, 2009) ponía delante del escenario a los principales intermediarios entre anunciantes y receptores de la publicidad. Quizás por primera vez, una cumbre sobre Cambio Climático se había convertido en un espacio político de primer orden y todos los agentes sociales querían estar presentes de una forma $u$ otra. En la presentación de la Campaña Hopenhague, el director de la IAA, Carlos Yepes, habló de "compromiso" y "responsabilidad" (Yepes, 2009). 
El imaginario medioambiental no es, desde luego, una novedad en la comunicación publicitaria. Durante décadas, las empresas anunciantes de diversos sectores han utilizado la fuerza de la iconografía medioambiental, a través normalmente de una idealización del espacio natural, con referencias a una abundante Naturaleza (extensos prados verdes, bosques frondosos, amplios cielos azules, etcétera). Mientras, los elementos humanos propios de la interactividad con el medio suelen quedar en un segundo plano. Así, la Naturaleza normalmente reflejada por la publicidad es una alegoría del jardín frondoso y, a veces, semi-salvaje, escenario abstracto que por su simplificación icónica es de fácil uso para la comunicación publicitaria.

Según el informe "Greenwashing Report" de la agencia TerraChoice, el numero de anuncios verdes se habría multiplicado por 20 en 10 años y triplicado desde 2006 (TerraChoice, 2011). La propia idea de Naturaleza es un elemento importante del discurso publicitario, como señalaba el estudio del MARM "Tratamiento del medio ambiente en la publicidad convencional (2006-2007)", donde entre los anuncios analizados que utilizan argumentos ambientales, el segundo atributo más recurrente es "natural". "Sostenible" es el cuarto atributo, tras "limpio" y con la mitad de presencia que el primero, "energía" (López Pastor et al., 2008). Los anunciantes han asociado sus productos a estos escenarios con el objetivo de reducir el sesgo industrial del producto, por ejemplo en sectores como el de la alimentación, donde el concepto "natural" es asociado a valores directos y positivos como la salud o el equilibrio emocional. Sin embargo, la introducción de ciertas tecnologías en el discurso publicitario ha supuesto la confluencia entre esta Naturaleza idealizada y la tecnología "limpia" como propuesta ante el reto de la sostenibilidad. Los molinos eólicos en el sector energético o el coche eléctrico en el de la automoción son un referente icónico de la producción y consumo sin impactos medioambientales. Así, la fuerza semántica de los aerogeneradores ha extendido su uso en multitud de materiales gráficos de empresas y entidades públicas pertenecientes a diversos sectores.

Este peso icónico de las tecnologías en sectores como la energía o la automoción viene a representar una interesante evolución gráfica donde el "verde naturaleza" ha ido pasando a un "azul tecnología limpia". Como resultado, las empresas automovilísticas proponen vehículos menos contaminantes y con menores emisiones de $\mathrm{CO}^{2}$, las energéticas proponen energías renovables, biocombustibles o mayor eficiencia energética, los grandes bancos proponen la inversión responsable, y todas proponen una nueva cultura empresarial donde confluyan en equilibro los ejes económico, social y medioambiental. El reclamo de lo "eco", lo "natural" y lo "sostenible" se hizo tan abundante en los años previos a la crisis de 2008, que el Ministerio de Medio Ambiente terminó creando un código de autorregulación para los grandes anunciantes, con el objetivo de que "no se abuse de la preocupación de los consumidores por el medio ambiente y no se explote la posible falta de conocimiento de los consumidores en materia ambiental" (MARM, 2009). De hecho, una investigación del Centro Complutense de Estudios de Información Medioambiental ya hablaba en 2012 de una creciente percepción de los argumentos en defensa del medioambiente como "presión institucional o marketing", e incluso de "ecofatiga ante los mensajes proambientales" (CCEIM, 2012).

\section{Ecofatiga en tiempos de crisis económica}

El activista medioambiental Jay Westerveld encontró ya a mediados de los 80 una de las claves de esta ecofatiga, y lo hizo en el cuarto de baño de un hotel. "Protege nuestro planeta: cada día, millones de litros de agua se utilizan para lavar toallas que sólo han sido utilizadas una vez", decía el cartelito que previene al huésped de usar en exceso las toallas de su habitación, como si no fuera también evidente, ironiza Westerveld, que el primer beneficiario de este movimiento "salvemos las toallas" (Motavalli, 2011) es el propio hotel. La misma lógica empresarial de ahorro de costes invita a los grandes bancos, por ejemplo, a sustituir la correspondencia bancaria por emails al cliente, pero se presenta comunicativamente como si se tratara de un gran esfuerzo de implicación de la entidad bancaria en la lucha por el medioambiente.

No hay duda de que es una estrategia inteligente. La empresa traslada la responsabilidad del reto en sostenibilidad al consumidor, que debe prescindir del servicio para no quedar éticamente señalado y, cuando a la entidad se le pide que tome la iniciativa y no ponga tantas toallas o que no las cambie cada día, responde que ya le gustaría, pero que es el consumidor el que no admite esos cambios porque no está lo suficientemente concienciado. Justamente, el desequilibrio entre lo rentable y barato que es hacer una campaña publicitaria 
diciendo lo responsable que es tu empresa, y los esfuerzos que requiere cambiar realmente el modelo de producción, es la clave de lo que Westerveld llamó "greenwashing" o lavado de imagen en materia de sostenibilidad medioambiental. "A medida que fue ganando terreno la «sensibilidad ambiental» de la población", explica Jose Manuel Naredo, "se observó que resultaba más fácil y ventajoso para políticos y empresarios contentarla a base de invertir en «imagen verde» que en tratar de reconvertir el metabolismo de la sociedad industrial y las reglas del juego económico que lo mueven" (Naredo, 2006).

Hoy es difícil que una gran empresa ya no cuente con un departamento específico de Responsabilidad Social Corporativa (RSC) o de su prima hermana, la Reputación Corporativa. A pesar de la crisis, que ha arrasado con muchos proyectos de RSC, sigue habiendo congresos y revistas especializadas en la temática y, sobre todo, decenas de rankings, premios y reconocimientos de todo tipo. Índices bursátiles como el Dow Jones Sustainability Index, listados de las empresas más sostenibles como el Anuario de PriceWaterhouseCoopers o premios como el European Business Award de Sostenibilidad Corporativa permiten a las grandes empresas presentarse como líderes en sostenibilidad. Estos premios, en su mayoría otorgados por consultoras privadas, se han convertido en un nuevo elemento de competencia empresarial, aupando a las "compañías líderes que ofrecen una inspiración crucial para aquellas que se encuentran en los niveles más bajos de la pirámide de sostenibilidad", dice el documento BluePrint del Pacto Global de la ONU. Y, sin embargo, esto nos lleva a la paradoja de la imagen proyectada: "A pesar de que se intenta desvincular la reputación de la estética de las buenas intenciones, sería pueril obviar que la imagen de marca obliga a las grandes compañías a tejer una reputación corporativa aceptable", reconoce un redactor de Corresponsables, una de las revistas especializadas en Responsabilidad Social Corporativa (Corresponsables, 2009). Y es que la RSC, a pesar de su rápido crecimiento como paradigma de una nueva cultura de la comunicación empresarial, sobrevive entre paradojas: si no es rentable (la dictadura del ROI, Retorno de la Inversión Publicitaria) no parece justificarse como una verdadera estrategia empresarial, y si es rentable, parece sólo una nueva estrategia comunicativa al servicio de las grandes multinacionales.

Conscientes de este problema de legitimación, la principal publicación de la Asociación de Directivos de Comunicación, DirCom, aconseja "evitar que la presentación de conductas responsables parezca propagandística o resulte incontrastable" (DirCom 2009). Pero no siempre es fácil. Por ejemplo, no es fácil dejar de pensar en Volkswagen y el escándalo de los motores trucados en sus coches para que puedan pasar los test de contaminación. Y tampoco es fácil olvidar Think Blue, una de las costosas campañas publicitarias de la marca alemana antes del escándalo del Diesel Gate, con el eslogan "Conservar lo verde es pensar en azul". Uno de los anuncios de la campaña mostraba un coche Volkswagen ante un enorme árbol: "Un árbol de serie. Lo último en tecnología alemana", decía el eslogan.

A pesar de la rápida institucionalización de la Responsabilidad Social Corporativa, no deja de ser llamativo que no existan casi herramientas para comprobar cuándo lo que dice una empresa es sólo un lavado de cara. Quizás porque lo que se obtiene de los pocos estudios que se han hecho tampoco invita al optimismo. Por ejemplo, la consultora TerraChoice ha realizado durante algunos años análisis sobre diversos productos del mercado. Así, de los 5.296 productos analizados en 2009, solo 265 cumplirían con los requisitos de sostenibilidad ambiental que su publicidad decía. Por su parte, el proyecto de investigación CSR IMPACT del programa Seventh Framework de la Unión Europea concluye que las políticas de Responsabilidad Social Corporativa no solo no suponen una contribución significativa a los objetivos de la Unión Europea sino que, además, su papel es mínimo en comparación con los enormes impactos sociales y medioambientales negativos o positivos- que las empresas europeas no contabilizan bajo la RSC (Bernard et al., 2013). Así que la Responsabilidad Social Corporativa vive en la cuerda floja, y el propio Carlos Sánchez Olea, vicepresidente de DirCom, dice en un artículo titulado "Esta RSC no emociona" que la RSC "pasa por momentos de confusión, y puede morir víctima de los excesos cometidos por el espectáculo creado por apóstoles sobrevenidos a esta nueva religión de la empresa, llamados a evangelizar por plazas y plateas a directivos, profesionales o políticos" (Sánchez Olea, 2009).

\section{En el patio trasero del supermercado}


Al final, el boom mediático del cambio climático no duró mucho. Tan sólo un año después de la repercusión alcanzada por la presentación del documental de AI Gore y los informes del IPCC explota la burbuja inmobiliaria y la inversión publicitaria retrocede 15 años, un 35\% (Infoadex, 2010). La crisis económica vendría acompañada de un enaltecimiento de todo el imaginario que rodea a la economía, los resultados empresariales y, sobre todo, el empleo. "La crisis llegó y, como no podía ser de otra forma, lo hizo acompañada de su propio discurso mediático. El desánimo, la desesperanza, la preocupación, el paro, la inflacción, las bolsas... se convierten en términos centrales del nuevo discurso que transmiten los medios de comunicación”, explica Martín Requero (2010). Mientras, el Barómetro del Centro de Investigaciones Sociológicas (CIS) constata un progresivo desinterés por las temáticas medioambientales, algo que también concluye el Observatorio de Cambio Climático y Medios de Comunicación (MECCO) de la Universidad de Colorado tras analizar la cobertura sobre este tema en 50 periódicos de todo el mundo (Andrews et al., 2016). Las temáticas medioambientales descienden en los discursos publicitarios [1] e incluso el fenómeno afecta a la dimensión comunicativa de la Responsabilidad Social Corporativa: "La intensa actividad de información acerca de la actual coyuntura económica por parte de los medios de prensa escrita ha restado protagonismo a la RSC", dice un estudio de Forética (2013).

Pero además, explica Pérez Ruiz (2011), bajo el nuevo prisma de la crisis surgen dudas sobre las motivaciones de los programas de RSC y de que se esté produciendo "una verdadera incorporación de los principios de la responsabilidad social a la identidad corporativa”. Otro estudio señala que la empresa es considerada por los consumidores como la fuente menos creíble para conocer sus propias actividades de RSC: "Los consumidores la perciben como una fuente interesada, oportunista y carente de credibilidad" (García Arrizabalaga et al., 2012). Y sin embargo, ese escepticismo, extensible a casi toda la comunicación que proviene de los grandes anunciantes, no parece servir de base para un tipo de consumo más crítico y transformador, dicen los sociólogos Luis Enrique Alonso, Carlos Jesús Fernández y Rafael Ibáñez: "el difícil contexto de la crisis, más que espolear conductas de consumo en las que se adopten filosofías alternativas en favor de la sostenibilidad, parece fortalecer las posiciones más reacias a la puesta en práctica de cambios personales y sociales que ayuden a transformar el modelo de consumo vigente" (Alonso et al., 2014).

Al final, por tanto, el paradigma que ha conseguido sobrevivir a todos estos vaivenes ha sido el del estante del supermercado, el discurso del consumo. "Se terminaron los grandes automóviles con grandes irresponsables consumos de gasolina, se acabaron las calefacciones de carbón y las chimeneas humeantes, al menos del paisaje más inmediato del ciudadano; ahora lo que viene es el consumo responsable, solidario, ecológico, pero no el fin del consumo", señalaba con ironía Raúl Eguizábal (2011). Por tanto, y a pesar del nuevo marco comunicativo generado por la RSC, la pregunta sigue intacta: ¿qué impactos sociales y medioambientales suponen los distintos modelos de producción?

Cada cierto tiempo recibimos indicios relacionados con esta pregunta, por ejemplo, cuando la prensa se hace eco del derrumbe de una precaria fábrica en la India y entre los escombros se hallan las etiquetas de las grandes marcas de ropa, o cuando en el telediario apenas se intuyen las calles de Pekin bajo una densa capa de niebla tóxica. De hecho, la Organización Mundial de la Salud (OMS) recuerda que más de 100.000 chinos mueren anualmente a causa de la contaminación del aire en sus ciudades. Es debido a las estufas de carbón y a los automóviles, pero también a las incontables fábricas que producen día y noche para una creciente clase media mundial que, según Brookings Institution (2017), alcanza ya los 3.000 millones de personas. Un estudio publicado por Energy Policy calcula que entre 2002 y 2008 , el $48 \%$ del total de las emisiones chinas estaban vinculadas a la producción de bienes para la exportación (Ming Xu et al., 2011).

La fábrica inglesa de la Revolución industrial teñía Londres con humo negro. La fábrica china del Siglo XXI genera una nube de contaminación que, en vez de funcionar como una cortina de humo, señala una de las fases clave en el origen de millones de productos destinados a los lineales del supermercado. Hace unos años, la marca Louis Vuitton mostraba en uno de sus anuncios a un artesano, pincel en mano, iluminación tenebrista y bigote velazquiano. En otro de la misma serie, la protagonista que cosía a mano un bolso era presentada como "la costurera, con hilo de lino y cera de abejas“. La Agencia de Regulación publicitaria inglesa (ASA) terminó retirando el anuncio por ofrecer una imagen irreal del modelo productivo de la marca. Esta construcción narrativa es una de las formas más potentes de desconexión, capaz no sólo de esconder cuando interesa la 
fábrica estandarizada, arquetipo del toyotismo, sino también capaz de sustituir la maquila asiática por un refinado taller renacentista. La verdadera producción artesanal de hoy en día, incluso entre las marcas de lujo, es la del modelo productivo que ha hecho de India, Corea, Indonesia o China las fábricas del Mundo.

No son los únicos patios traseros de la sociedad de consumo. Solo hay que recordar todos los conflictos alrededor del coltán africano para comprender que más allá de la fábrica desconectada está el punto de origen de las distintas materias primas. "La población privilegiada de América del Norte y Europa", dice Naomi Klein, "podía, mediante prodigios de negacionismo diversos, aislar mentalmente esos emplazamientos desafortunados situándolos dentro de la categoría de patios traseros, eriales, no lugares o, en el supuesto más desgraciado de todos, sitios perdidos en medio de ninguna parte" (Klein, 2015:382). A este negacionismo, que tanto tiene que ver con desconectar por sistema el consumo de su forma de producción, Klein se refiere como "el gran pacto de la era del carbono": "las personas que se aprovechan de las ventajas del extractivismo fingen no ver los costes de ese confort siempre y cuando las zonas de sacrificio sean eficazmente mantenidas fuera del campo de visión" (Klein, 2015:382).

\section{Las cuentas de la energía low cost}

Dice Thomas Piketty en "El capital en el siglo XXI" que los asuntos económicos son algo demasiado importante como para dejárselos solo a los economistas y que, por eso mismo, las cifras y los números se convierten en herramientas clave para la ciudadanía (Piketty, 2014:649). Efectivamente, los números que arroja la trazabilidad del producto pueden ayudarnos a entender un modelo de producción y consumo con tantas zonas en penumbra. Por ejemplo, comparando el precio de una camiseta de algodón en un enorme establecimiento Primark y los costes económicos asociados a los procesos de producción, transporte y venta de este tipo de producto: ¿tan sumamente poco ha costado extraer la materia prima de la camiseta, fabricarla, transportarla de un lado a otro del mundo y ponerla a la venta, para que a alguien le sea rentable vender una camiseta por un euro? ¿En qué consiste el milagro?

Ya sabemos que la mano de obra barata y desregulada en los países productores y los sueldos precarios en los países consumidores permiten reducir enormemente el coste final del producto. Pero hay otros factores clave, y uno de ellos es especialmente importante porque está presente en todas las fases de extracción, fabricación y distribución del producto. La industria textil, explica Enric Carrera, consume combustibles fósiles para el funcionamiento de la maquinaria agrícola utilizada en los procesos de obtención de las fibras naturales. Usa energía eléctrica, generada a partir de los combustibles fósiles, para el funcionamiento de la maquinaria industrial utilizada en el largo proceso textil (obtención de fibras químicas, hilatura, tisaje, ennoblecimiento y confección). Y a esto hay que sumarle la gasolina y gasoil empleados para mover materias primas y mercancía de un sitio para otro.

Efectivamente, el milagro es la energía fósil. Pero en unas condiciones determinadas. La constitución material de la sociedad de consumo y de la clase media, explica Nathan John Hagens en el informe de The Worldwatch Institute (2015), ha sido el resultado de introducir energía a tareas que se realizaban manualmente, como labrar la tierra o manufacturar, creando una multitud de aparatos y artefactos "voraces en consumo de energía". Entre 1850 y 2010, explica Hagens (2015), la población humana se multiplicó por 5, pero el consumo energético mundial se multiplicó por 20 y el de combustibles fósiles por 150. "La energía fósil barata es el fundamento de nuestras ganancias, de nuestros sueldos elevados y de unos bienes y servicios de bajo coste", señala Hagens. La relativa facilidad para explotar en los últimos cien años los yacimientos de petróleo, debe entenderse como una excepción en la relación entre las distintas sociedades y la energía. Durante este históricamente breve pero intenso episodio, cada unidad de trabajo humano es respaldada en la economía industrializada por unas 360 unidades de trabajo alimentado con energías fósiles (Hagens, 2015:45).

Como resultado, tras la Segunda Guerra Mundial comienza a desplegarse una asombrosa colección de objetos de consumo, y los electrodomésticos y los nuevos productos para la naciente clase media presentan incluso una dimensión lingüística, señala Alonso (2006): "El consumo entra en un orden general de las significaciones en el que las dimensiones simbólicas tienden a recurrir y totalizar cualquier práctica adquisitiva". "En la 
Enciclopedia", diría Baudrillard (1968) a finales de los sesenta, "el hombre pudo ofrecer un cuadro completo de los objetos prácticos y técnicos de que estaba rodeado. Después se rompió el equilibrio: los objetos cotidianos (no hablo de máquinas) proliferan, las necesidades se multiplican, la producción acelera su nacimiento y su muerte, nos falta un vocabulario para nombrarlos".

Aunque la primera clase media no es global, el modelo que la sustenta prácticamente lo es, así que la energía y el transporte, dos de los sectores clave en las emisiones de gases GEI, abanderan desde hace décadas el ranking de los contribuyentes al calentamiento global. "Con la implantación del sistema del libre comercio internacional y de la producción deslocalizada como norma, las emisiones no solo trasladaron su foco de unos países a otros, sino que se multiplicaron", explica Naomi Klein (2015). El propio IPCC, dice Klein, ha tenido que reconocer por fin en su Quinto informe de Evaluación que "una proporción creciente de las emisiones antropogénicas totales de $\mathrm{CO} 2$ se liberan en la fabricación de productos que compran y venden en el comercio transfronterizo internacional".

En los inicios del proceso industrializador, nos recuerdan Peter A. Victor y Tim Jackson, la contaminación de los ríos y del aire de las ciudades, o la sobrecarga de los vertederos municipales, son problemas principalmente locales, "pero la expansión económica global hizo que los problemas ambientales pasaran a ser regionales (por ejemplo, la lluvia ácida, el transporte y eliminación de residuos peligrosos) y posteriormente globales (por ejemplo, la acidificación de los océanos, la pérdida de biodiversidad y el cambio climático)" (The WorldWatch Institute, 2015).

\section{El futuro perfecto}

Uno de los últimos anuncios de Caja Madrid, antes de pasar a formar parte de Bankia, mostraba a una joven surfista en una solitaria playa. Hasta ahí todo normal, dentro del discurso publicitario que ubica la acción en espacios evocadores y románticos. Pero la protagonista está jugando con un perro robot futurista, que lleva en la boca billetes de 50 euros: "Futuro Perfecto", titula el anuncio, y prosigue: "Invertimos 3000 millones de euros en tecnología para que la banca del mañana sea la que todos queremos. Plan tecnológico Caja Madrid”. ¿Será así el futuro? La clase media de no más de 1.000 millones de consumidores que generaba en los años 70 el primer aumento importante de las emisiones GEI, dentro de 15 años será una clase media formada por 5.300 millones de personas, señala The Brookings Institution (2017).

La economía ecológica considera que, en ese escenario, el aumento global en la demanda de recursos que llevará aparejado el seguir por la senda del crecimiento, superará inexorablemente los beneficios medioambientales conseguidos a través de la eficiencia y la tecnología. Antes bien, que ese discurso de la eficiencia tecnológica no solo no parece resolver el escenario que los científicos del IPCC presentan, sino que incluso parece generar el efecto contrario, prorrogando enfrentarse a los verdaderos problemas de la sostenibilidad. "Una mayoría de economistas y de gobiernos", dicen Peter A. Victor y Tim Jackson, "se resiste a abordar las implicaciones del crecimiento económico para la biosfera, y prefiere aferrase a la esperanza de un desacoplamiento absoluto, conseguido mediante una combinación de cambios tecnológicos y la apuesta por una economía más basada en los servicios. Ambas vías de cambio son importantes. Pero la evidencia existente sugiere que es improbable un desacoplamiento absoluto del crecimiento económico y del trasiego de materiales y energía" (The WorldWatch Institute, 2015:80).

La desconexión entre el escaparate de la tienda y el patio trasero de la producción, elemento constitutivo de la sociedad de consumo para la gran clase media global, renace en el mito del desacoplamiento absoluto entre el crecimiento económico y sus requerimientos e impactos materiales. Pero esta vez, la clave está en la energía y el optimismo tecnológico. "Líder mundial en la lucha contra el cambio climático", decía la última campaña de Iberdrola, a pesar de pertenecer a un sector que es el causante de dos tercios de las emisiones GEI a nivel global. De hecho, la enorme proyección mediática de este sector, señala Mercado Saez (2016), ayuda a "desmaterializar el problema", es decir, desconectar el cambio climático de sus verdaderas causas y, por tanto, a "dificultar la acción de los ciudadanos, no sólo en cuanto al cambio de hábitos o comportamientos en relación al consumo, sino, sobre todo, en cuanto al incremento del nivel de exigencia a los representantes políticos, los 
que, al fin y al cabo, han de tomar las decisiones" (Mercado Saez, 2016).

En el supermercado comienza a borrarse la linea de trazabilidad del producto y con ella, los impactos medioambientales generados por el modelo de producción. La incapacidad para cuestionar el crecimiento y lograr ubicarlo en el lado de las causas en lugar del de las soluciones está detrás del hecho de que no se estén planteando medidas que vayan más allá de la tecnología, la eficiencia y, sobre todo, el consumo, cuando la inmensa presión sobre los recursos naturales y el propio cambio climático parecen aconsejar el camino contrario. Mientras, hablaremos del tiempo.

\section{Bibliografía}

Acciona (2007). "La campaña SOStenibilidad.com de ACCIONA se exhibirá en el Museo Reina Sofía". Comunicado, 15 de junio de 2007.

Alonso, L. E.; Fernández Rodríguez, C. J. y lbáñez Rojo, R. (2014). "Crisis y nuevos patrones de consumo: discursos sociales acerca del consumo ecológico en el ámbito de las grandes ciudades españolas", Empiria, 29, pp. 13-38.

Alonso, L. E. y Fernández Rodríguez, C. J. (2014). Los discursos del presente. Madrid: Siglo XXI.

Alonso, L. E. (2006). La era del consumo. Madrid: Siglo XXI.

Andrews, K., Boykoff, M., Daly, M., Gifford, L., Luedecke, G., McAllister, L., and Nacu-Schmidt, A. (2016). World Newspaper Coverage of Climate Change or Global Warming, 2004-2016. Center for Science and Technology Policy Research, Cooperative Institute for Research in Environmental Sciences, Universidad de Colorado.

ASPRIMA-SIMA (2007). Entrevista a Isabel Antúnez. Madrid: ASPRIMA-SIMA.

Azuero, D. (2009). La comunicación de la RSE. Propuestas para un modelo de comunicación responsable. Madrid: Forética.

Baudrillard, J. (1968). Le système des objets. París: Les Éditions Gallimard.

Bernard, B.; Kluge, T.; Schramm, E. y Schultz, I. (2013). Impact Measurement and Performance Analysis of CSR (IMPACT). Bruselas: UE, Institut for Social-Ecological Research.

Cáceres, P. (2007). “¿Qué es el IPCC?”, El Mundo, NATURA n¹4, 12 de mayo de 2007.

Cardona, J. (2009). Entrevista. Compromiso RSE n¹. Barcelona: Custommedia

CCEIM (2012). “Cambio Global en España 2020/50: Consumo y estilos de vida”, CCEIM, Barcelona: CCEIM.

CEACCU (2009). Análisis del etiquetado de los alimentos. La información obligatoria y nutricional en las etiquetas. Madrid: CEACCU.

Coll Rubio, P. (2016). "Nuevas tendencias en la publicidad en el punto de venta", Marketing+ventas, n. ${ }^{\circ} 305$, marzo/abril de 2016, pp 48-49.

Comisión Europea (2005). "Estrategia temática sobre el uso sostenible de los recursos naturales”. Bruselas: CE Comisión Europea (2011). "A resource-efficient Europe - Flagship initiative under the Europe 2020 Strategy". Bruselas: CE

Comisión Europea (2011). "Iniciativa emblemática para una Europa eficiente en recursos”. Bruselas: UE.

Corrales Crespo, P. (2000). "El lugar común en la construcción e interpretación del texto publicitario", Círculo de Lingüística Aplicada a la Comunicación 1, 13-27. Madrid: Universidad Complutense de Madrid. 
Correa, R.I.; Guzmán, M.D. y Aguaded, J.I. (2000). La mujer invisible. Huelva: Grupo Comunicar.

Corresponsables (2009). Editorial. Revista Corresponsables, 20.

Coseriu, E. (1992). Competencia lingüística: elementos de la teoría del hablar. Madrid: Gredos.

DirCom (2009). La comunicación responsable, clave para el fomento de la RSE. Barcelona: Mediaresponsable.

EUFIC (2011). Nutrition labelling. Nota de prensa, 24 de noviembre de 2011.

Eguizábal Maza, R. (2011). El estado del malestar: Capitalismo tecnológico y poder sentimental. Barcelona: Península.

Ezquerra Martínez, Á. y Fernández-Sánchez, B. (2014). "Análisis del contenido científico de la publicidad en la prensa escrita”, Revista Eureka sobre Enseñanza y Divulgación de las Ciencias, 11(3), pp. 275-289.

Fernández Buey, F. (2006). En Jorge Riechmann, Biomímesis. Ensayos sobre imitación de la naturaleza, ecosocialismo y autocontención. Madrid: Catarata.

Fernández Durán, R. (1993). La explosión del desorden: la metrópoli como espacio de la crisis global. Madrid: Fundamentos.

Forética (2013). I“RSE en los medios, el reto de una comunicación más interactiva”, Madrid: Forética.

Gaja i Díaz, F. (2005). Revolución informacional, crisis ecológica y urbanismo. Valencia: Ed. Univ. Politécnica.

García Arrizabalaga, I., Gibaja Martíns, J.J. y Mujika Alberdi, A. (2012). Credibilidad de las fuentes de información sobre responsabilidad social corporativa. Revista de Responsabilidad Social de la Empresa, 4 (1), pp. 59-78.

IAA (2009). Misión. [Fecha de consulta:18/6/2011]. http://espanol.hopenhagen.org/mission

Iranzo, J.M. (2008). “Camino a Bali: Cambio Climático y cambio social global”, Nómadas. Revista Crítica de Ciencias Sociales y Jurídicas $n^{\circ} 17$.

IRP International Resource Pannel (2011). "Decoupling natural resource use and environmental impacts from economic growth". Bruselas: UNEP

Jenkins, J., Nordhaus, T. y Shellenberger, M. (2011). "Energy Emergence. Rebound \& Backfire As Emergent Phenomena". Breakthrough Institute.

Klein, N. (2015). Esto lo cambia todo. El capitalismo contra el clima, Barcelona: Paidós.

Lizcano, J.L. (2009). "Hacia una normalización de la información corporativa. El reporte de RSC y los sistemas de verificación”. Cuaderno Telos, 79.

López Pastor, A.T.; De Andrés del Campo, S.; González Martín, R. (2008). Tratamiento del medio ambiente en la publicidad convencional (2006-2007) Valladolid: Universidad de Valladolid y Ministerio de Medio Ambiente, Medio Rural y Marino.

Madrid Cánovas, S. (2005). Semiótica del discurso publicitario: Del signo a la imagen. Murcia: Universidad de Murcia.

MARM (2009). Código de autoregulación sobre argumentos ambientales en comunicaciones comerciales. Madrid: MARM.

Martín Requero, M. I. (2010). La publicidad social audiovisual: fines y formas. En Susana de Andrés del Campo (coord.). Otros fines de la publicidad, pp. 13-46. Sevilla: Comunicación Social. 
Mercado Saez, M. T. (2016). "Cambio climático y energía, una relación desdibujada en la prensa española", Redes.com n¹3, pp. 56-71.

Ming Xu et al. (2011). "CO2 emissions emboiled in China's exports from 2002 to 2008: a structural decomposition analysis", Energy Policy, 39, pp. 73-83.

MITyC (2009). "RSE Y PYME. Del discurso a la implementación”. Madrid: Ministerio de Industria, Turismo y Comercio.

Motavalli, J. (2011). “A History of Greenwashing: How Dirty Towels Impacted the Green Movement”. AOL Daily Finance.

Myers, G. (1994). Words un Ads, Nueva York: E. Arnold.

Naredo, J.M. (1996). Sobre el origen, el uso y el contenido del término sostenible. Primer catálogo español de buenas prácticas. Madrid: Ministerio de Obras Publicas, Transportes y Medio Ambiente.

Naredo, J.M. (2006). Raíces Económicas del Deteriorio Ecológico y Social. Madrid: Siglo XXI.

Hagens, N.J. (2015). “Energía, deuda y el fin del crecimiento”. En The WorldWatch Institute. La situación del mundo 2015.

OSE (2009). Cambio Global España 2020/50. Programa ciudades. Hacia un pacto de las ciudades españolas ante el cambio global. Centro Complutense de Estudios e Información Medioambiental, Madrid: Fundación CONAMA y Observatorio de la Sostenibilidad (OSE).

OSE (2015). Responsabilidad de las grandes empresas energéticas e industriales de España en el cambio climatico . Madrid: Observatorio de la Sostenibilidad (OSE).

Piketty, T. (2014). El Capital en el siglo XXI. Madrid: FCE España (Original en francés, 2013).

Pedrós Pérez, G. y Martínez-Jiménez, P. (2010). "Publicidad, educacion ambiental y calentamiento global". En Heras, F. et al. (coord.). Educación ambiental y cambio climático: Respuestas desde la comunicación, educación y participación ambiental (pp.103-120). Santiago de Compostela: CEIDA.

Pérez Ruiz, A. (2011). Estudio de la imagen de responsabilidad social corporativa: formación e integración en el comportamiento del usuario de servicios financieros. Tesis doctoral, Universidad de Cantabria.

Riechmann, J. (2006). Biomímesis. Ensayos sobre imitación de la naturaleza, ecosocialismo y autocontención. Madrid: Catarata.

Riechmann, J. (1995). “Desarrollo sostenible: la lucha por la interpretación”. En Riechmann, J. et al. (coord.). De la economía a la ecología. Madrid: Trotta.

Taibo, C. (2009). En defensa del decrecimiento: Sobre capitalismo, crisis y barbarie. Madrid: Los Libros de la Catarata.

TerraChoice (2011). Greenwashing Report 2010. [Fecha de consulta: 12/11/2015]. http://sinsofgreenwashing.org

The Brookings Institution (2017). The unprecedented expansion of the global middle class. An update. [Fecha de consulta:8/6/2017] https://www.brookings.edu/wp-content/uploads/2017/02/global_20170228_global-middleclass.pdf

The WorldWatch Institute (2015). La situación del mundo 2015. Barcelona: Icaria.

Torras, J. (2009). Entrevista de V. Adell. Compromiso RSE n¹. Barcelona: Custommedia.

Tramullas, M. A. (2016) "El cambio climático logra poner de acuerdo al mundo: hay que actuar", El Economista, 2 de noviembre de 2016. 
UICN Red List (2007), "Species extinction. The facts". Species Survival Commission. [Fecha de consulta: 12/3/2012]. http://cmsdata.iucn.org/downloads/species_extinction_05_2007.pdf

UIP, United International Pictures (2006). "Cómo se hizo "Una verdad incómoda”, Material promocional. NewYork: UIP.

Vilarnovo, A. (2005). En Romero Gualda, M.V. (coord.) Lenguaje publicitario: la seducción permanente. Barcelona: Editorial Ariel.

WWF (2010). "Living Planet Report 2010. Biodiversity, biocapacity and development". [Fecha de consulta:21/7/2013]. http://awsassets.panda.org/downloads/lpr2010.pdf

Yepes, C. (2009). "Zapatero, entre los líderes mundiales que acudirá a la Cumbre del Clima”, Europa Press, 25 de noviembre 2009.

[1] Véase el trabajo de Pedrós Pérez, G. y Martínez-Jiménez, P. (2010). Publicidad, educacion ambiental y calentamiento global. En: Heras, F. et al. (coord.). Educación ambiental y cambio climático: Respuestas desde la comunicación, educación y participación ambiental (pp.103-120). Santiago de Compostela: CEIDA.

- Recibido: 16 de junio de 2017

- Aceptado: 20 de junio de 2017

Ámbitos. Revista Internacional de Comunicación, n.37, edición de verano, 2017. 